\title{
Analysis of Supervised Machine Learning Techniques for Diagnosis of Disease of Infant Baby
}

\author{
Avali Banerjee | Shobhandeb Paul| Santanu Saha I Suvasish Paul \\ Department of ECE, Guru Nanak Institute of Technology, Kolkata, West Bengal, India
}

To Cite this Article

Avali Banerjee,; Shobhandeb Paul,; Santanu Saha \& Suvasish Paul. Analysis of Supervised Machine Learning Techniques for Diagnosis of Disease of Infant Baby. International Journal for Modern Trends in Science and Technology 7, 4-9 (2021).

\section{Article Info}

Received on 27-April-2021, Revised on 20-May-2021, Accepted on 24-May-2021, Published on 01-June-2021.

\section{ABSTRACT}

A system for monitoring an infant's health is developed and described in this paper. In this system, smoke detector, sound sensor, temperature and humidity sensor, are interfaced with the controller Node MCU-ESP8266. In the system, ThingSpeak Cloud is used for the data processing. ThingSpeak Cloud is connected to the Wi-Fi based microcontroller. The behavior and the problems that are being detected can be easily notified to the parents apart from the doctors and nurses, So, that even the nurses or the doctors misses out by chance, the parents can handle the scenario. The collected data can be taken out as in the form of the csv format. This data can be easily put into the Machine Learning Model in order to predict the various problems that a baby might be suffering from. These predictions have been done solely upon the data collected from the individual baby. Furthermore, separate system-based report would be facilitated by the model itself

KEYWORDS: AI, BP Series, Controller, Health Monitoring System, IFTTT, IOT, Pulse Sensor, ThingSpeak

\section{INTRODUCTION}

In 19 th century there was suddenly a huge transformation in the human lives. Everything just changed from tip to toe within few years. The standard of living has been changed as compared to the previous centuries. Basically, that was the mark for the "Modern Era" of the human lives. And for modern era the requirement is to produce more in less amount of time. This gave birth to the industries, viz., food, clothing, transportation, medical industry, etc. Food and clothing industries are very essential for the huge growing population. Similarly, transportation industry is also very important for faster traveling from one place to another. These industries made their marks and still today these industries are progressing in such a way for the humans making their lives smooth and easy. Technologies has been advanced in the 21 st century than what was there in the 19th century.

In 21 st century, we have the concept of "Embedded Systems and IOT-Internet of Things". In this century, the development of all the industries depends upon the technological advancements of the electronics field. Today, there is no such industries left where there is no minimal use of the electronics devices. We can see electronic devices everywhere. "Embedded Systems", are basically the electronics, electrical or the mechanical devices that we use in our day to day life. Different embedded interfacings can be done but when it is connected to the internet then it is known as Internet of Things. IOT applications in the present era is very huge.

This paper basically aims on the above-mentioned concept of IOT. Today, there is also a huge boom of IOT applications in the medical industry. But, still there are some or the other 
mishaps that takes place in here. This paper describes a baby monitoring system which will help the parents to ensure the proper care and safety of their babies. This work basically aims on those infant babies, who are needs critical care under the doctors and nurses. It is fact that each doctor and nurse have minimum of 20-30 infant babies under their observation so sometimes there is a possibility of missing out a baby's condition.

There are many existing monitoring systems that almost resembles same as this monitoring system, but the way it is different from other monitoring systems is that rather than providing the instant alarm, it also keeps records of a particular baby as a document format for future references and moreover this is the cheapest form of the monitoring system.

\section{Circuit Diagram}

\section{A. NodeMCU1.0 (ESP-12E Module)}

NodeMCU popularly known as the Wi-Fi module which is used in various fields of IOT and this device application is on a large scale.

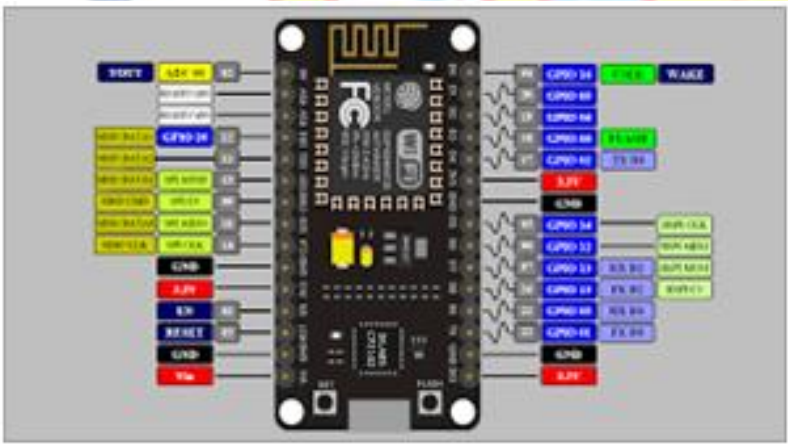

Fig. 1. NodeMCU 1.0 (ESP-12E Module)

The Wi-fi module is fitted with the chipset, whose model no. is ESP8266. There are basically 30 pins in the board to be used in any functions according to the user. The specifications of the pins are described below:

\begin{tabular}{|l|l|}
\hline Microcontroller & ESP8266 \\
\hline Frequency & $80 \mathrm{MHz}$ \\
\hline Flash & $4 \mathrm{MB}$ \\
\hline RAM & $80 \mathrm{~KB}$ \\
\hline Vendor & NodeMCU \\
\hline
\end{tabular}

digital pin and $1+\mathrm{VCC}$ and GND pins. Also, there is a LED fitted in the module that dims or lights up whenever there is a peak sound detected or low sound detected.

Sound is detected through microphone and fed to the LM393 op amp. The working voltage of the sound sensor is: DC $3.3-5 \mathrm{~V}$ and the dimensions of the sensor is: $45 \times 17 \times 9 \mathrm{~mm}$.

There are various types of sound sensors, the users can choose their choice of sensor according to their field of applications as various sound sensors detect various ranges of sounds.

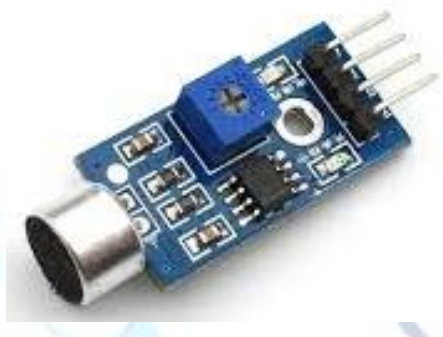

Fig. 2. Sound sensor (RKI-3103)

\section{DHT-11-Temperature-Humidity Sensor}

The DHT-11 or the temperature and the humidity sensor is the electronic device or rather the module that can detect the humidity and the temperature at the given time and place.

The main specifications of the DHT- 11 are given below:

\begin{tabular}{|l|l|}
\hline Resolution & $1^{\circ} \mathrm{C}$ \\
\hline Accuracy & $\pm 2^{\circ} \mathrm{C}$ \\
\hline Measuring range & $0^{\circ} \mathrm{C} \sim 50^{\circ} \mathrm{C}$ \\
\hline Humidity Resolution & $1 \% \mathrm{RH}$ \\
\hline Accuracy & $\pm 5 \% \mathrm{RH}\left(0 \sim 50^{\circ} \mathrm{C}\right)$ \\
\hline Measuring range & $\begin{array}{c}20 \% \mathrm{RH} \sim 90 \% \mathrm{RH} \\
\left(25^{\circ} \mathrm{C}\right)\end{array}$ \\
\hline Operating voltage & $3.3 \mathrm{~V} \sim 5.5 \mathrm{~V}$ \\
\hline $\begin{array}{l}\text { Recommended } \\
\text { storage condition } \\
\text { Temperature }\end{array}$ & $10^{\circ} \mathrm{C} \sim 40^{\circ} \mathrm{C}$ \\
\hline Humidity & $60 \% \mathrm{RH}$ or below \\
\hline
\end{tabular}

As this is a temperature and the humidity sensor, it has vast applications in Weather Stations, Humidity control monitoring and many other areas of such kind.

\section{B. Sound Sensor}

The sound sensor is also basically a electronic device rather a module wherein all the capacitors and the resistors connections are made. A typical sound sensor consists of 4 pins -1 analog and 


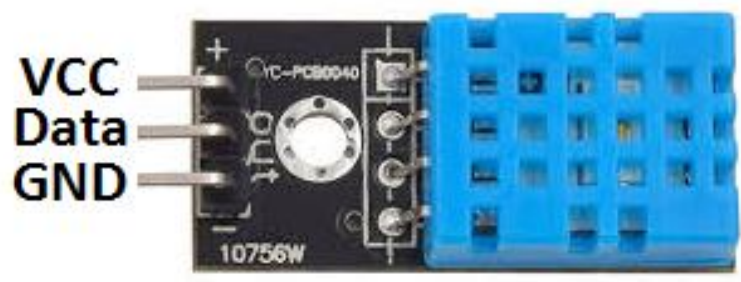

Fig. 3. DHT-11 Temperature-Humidity sensor

\section{Arduino-IDE}

The Arduino-IDE is the open source IDE (Integrated Development Environment) which gives user the platform to write, compile the program and upload and run the same to the board. The GUI provided by the Arduino-IDE is very easy to understand or rather a very user-friendly platform for the users to code on.

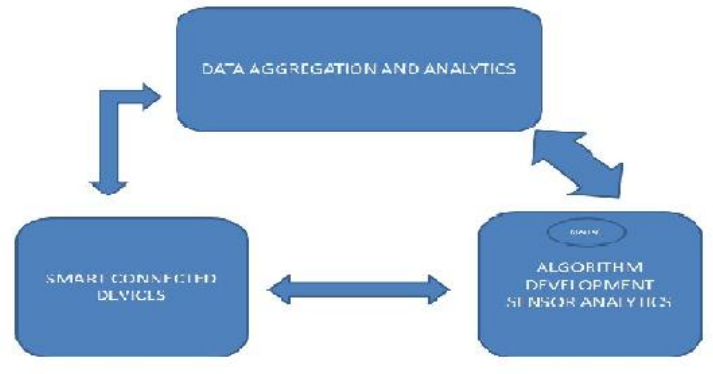

Fig. 4. ThingSpeak

\section{E. ThingSpeak}

ThingSpeak is an open-source IOT platform that provides the user a variety of choices so that the users can put a lot to their task and apart from this it is also a very user-friendly platform for the users to work on. It is a platform wherein the user can get the real time values of the sensors detect. This platform can also be used to fetch the data and trigger via IFTTT can help in performing various interesting operations.

\section{F. IFTTT}

IFTTT is basically a freeware online cloud service that enables the user to manipulate as well as perform some conditional statements. This particular platform provides the user many app services that the users can put their use into, and the good part of this cloud service is it is a very user-friendly platform.

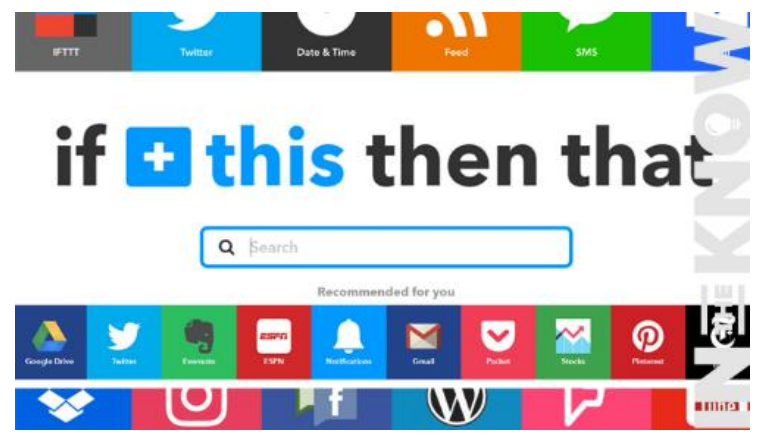

Fig. 5. IFTTT app

\section{G. Machine Learning}

Basically, there are two methods to approach a Machine Learning model. Supervised and Unsupervised learning, out of which we have used the Supervised Learning, to achieve our required target.

Supervised learning provides a tool to classify and process data. It uses machine learning. It is learning a function that maps an input to an expected output. It refers a function from labeled training data consisting of a set of training example.

\section{H. Data Analysis}

We needed data at first and as mentioned we got the data from the smart sensors, on the existing dataset, feature engineering was done and then the model was trained using the purposed algorithm and the best accuracy was obtained. The snippet of the dataset used is given below:

\begin{tabular}{lrrrrrrrr} 
& \multicolumn{2}{r}{ created_at } & Unnamed: 1 & Temp & Humidity & Moisture & Sound & Status \\
\hline $\mathbf{0}$ & $2019-07-22$ & $16: 53: 47$ IST & NaN & 28.5 & 77.0 & 16.0 & 14.0 & Fever \\
1 & $2019-07-22$ & $16: 54: 03$ IST & NaN & 27.0 & 76.0 & 16.0 & 14.0 & Stomach Pain \\
2 & $2019-07-22$ & $16: 54: 19$ IST & NaN & 27.0 & 76.0 & 16.0 & 14.0 & Common Cold \\
3 & $2019-07-22$ & $16: 54: 35$ IST & NaN & 27.0 & 77.0 & 16.0 & 14.0 & Food Poisoning \\
4 & $2019-07-22$ & $16: 54: 52$ IST & NaN & 27.0 & 76.0 & 16.0 & 14.0 & Influenza (flu)
\end{tabular}

Fig. 6. Dataset

\section{K-Nearest Neighbour}

K - Nearest Neighbor Classification is a closestneighborclassifier depend on the possibility that an object ought to be anticipated to have a place with a similar class as the items in the preparation set with the greatest likeness.

It does not assume about the distribution of data as it is non-parametric.

We have taken training data which classifies coordinates into groups and are identified by an attribute. 


\section{Results}

Fig. 7(a) represents the cut-out of the graph that is been fetched from the temperature and the humidity-sensor and here the temperature collected data is displayed via graphical representation by ThingSpeak. From the graph shown, we can see the real-time value of the temperature that had been taken during the testing time.

Fig. 7 (b) the cut-out of the graph that is been fetched from the sound-sensor and displayed via graphical representation by ThingSpeak. the graph shown, we can see the real-time value of the temperature that had been taken during the testing time.

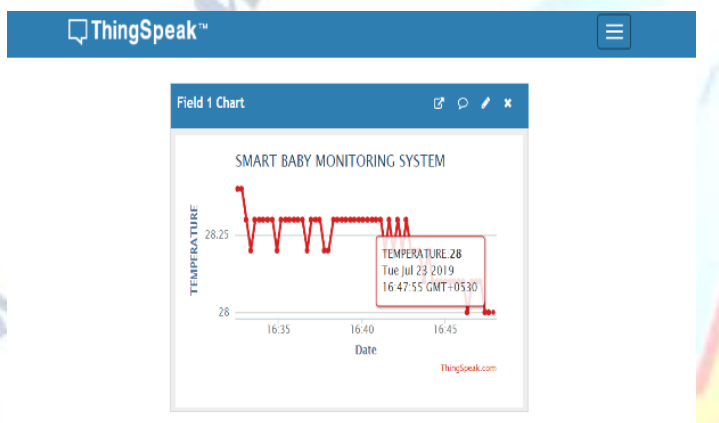

Fig.7 (a). Real-time temperature data during testing time

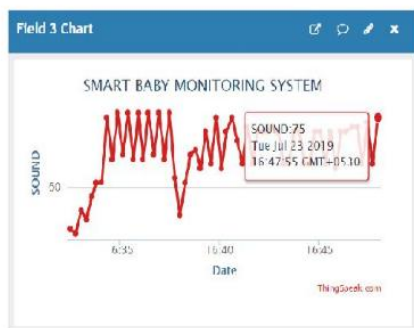

Fig. 7(b) Real-time sound data during testing time

Fig. 7(c) represents the cut-out of the graph that is been fetched from the temperature and the humidity-sensor and here the humidity collected data is displayed via graphical representation by ThingSpeak. From the graph shown, we can see the real-time value of the temperature that had been taken during the testing time.

Fig. 8 shows the circuit of baby monitoring system. First of all the dataset has been visualized and then proceeded further for the result. The data visualization is shown in Fig. 9.

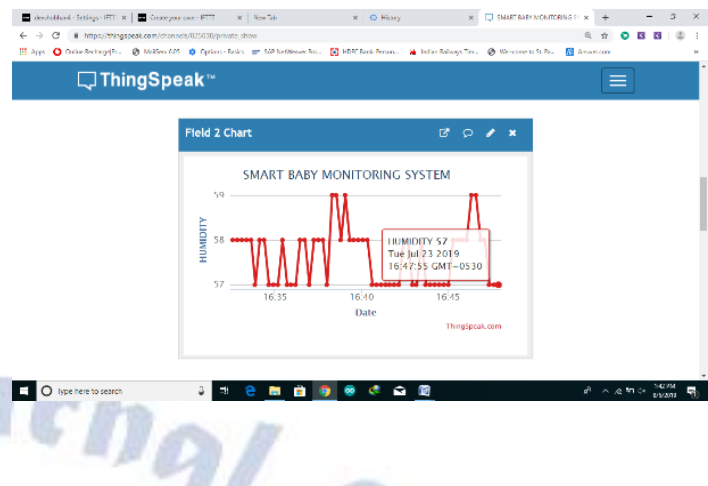

Fig. 7(c) Real-time humidity data during testing time

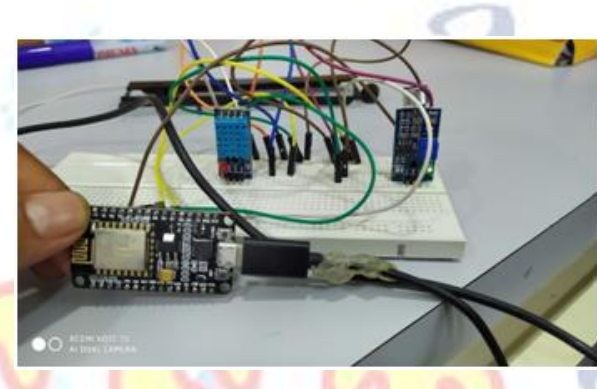

Fig. 8. Baby monitoring system

For the data visualization and plotting we have used the Matplotlib and the Seaborn, which is based on the Python data visualization library based on Matplotlib. It provides a high-level interface for drawing attractive and informative statistical graphics.
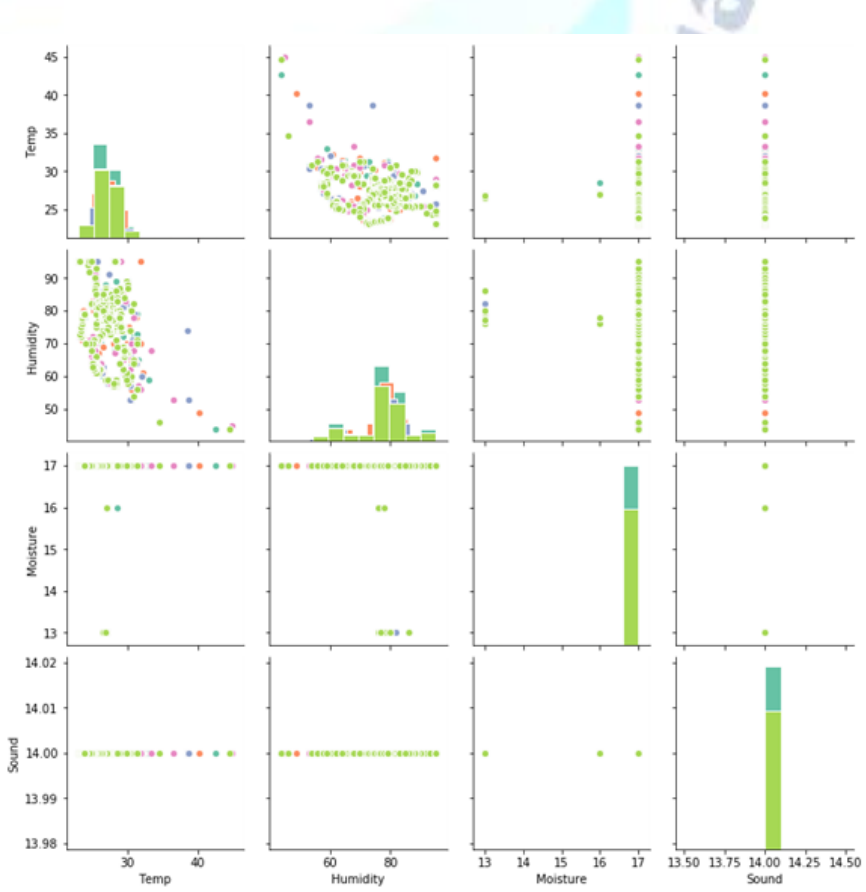

Fig. 9. Data Visualization 


\section{A. Evaluation Method}

True Positive (TP): Cases when classifier predicted TRUE (they have the disease), and correct class was TRUE (patient has a disease).

True Negative (TN): Cases when classifier predicted FALSE (no disease), and correct class was FALSE (patient do not have disease).

False Positives (FP) (Type 1 error): Cases when classifier predicted TRUE (they have the disease), and correct class was FALSE (patient do not have disease).

False Negative (FN) (Type 2 error): Cases when classifier predicted FALSE (no disease), but they actually and correct class was TRUE (patient do have disease).

$$
\text { Classification accuracy }=\frac{(T P+T N)}{(T P+T N+F P+F N)}
$$

Misclassification rate (Error rate)

$$
=\frac{(F P+F N)}{(T P+T N+F P+F N)}
$$

$$
\text { Precision }=\frac{T P}{\text { TotalTruePredictions }}=\frac{T P}{(T P+F P)}
$$

$$
\text { Recall }=\frac{T P}{\text { ActualTrue }}=\frac{T P}{(F N+F P)}
$$

The values of the TP, TN, FP, and $\mathrm{FN}$ can be collected from the heat map and can be substituted to get the table for precision, recall, f1-score and support (Misclassification rate).

Classification models in machine learning algorithm were tested to make the best use of the clinical data provided online to be able to predict the dataset.

\section{B. Result from the Model}

y_pred_val

array (['Influenza (flu)'], dtype=object)

In order to achieve this result, we have used Intel i3 generation processor with 4Gb RAM \& Ubuntu OS. 3.7.0 Python version used with Jupyter notebook IDE tool. For analysis of this machine learning model we have used the confusion matrix showing the calculation of the precision, recall, f1-score.

\section{Conclusion}

The smart baby monitoring system can be used to provide information accurately, directly to parents, person who takes care of the infant baby, i.e., the doctors and the nurses. In e-health monitoring system, a framework for health systems provides service-oriented architecture. This framework should have a high flexibility and extensibility. By using this framework, the doctors and parents can monitor alteration in body temperature and heart rate of child accurately. So, it will be possible for them to analyze the physical characteristics of child and take the decisions accordingly.

\section{SCOPE FOR THE FUTURE}

A better version of it can be made that can detect the position of the baby and the same can be notified via cloud to the parent's device. Also, it is seen that the pattern in which the baby cries indicates the type of problem the baby is suffering from, so this can be improved by using suitable devices.

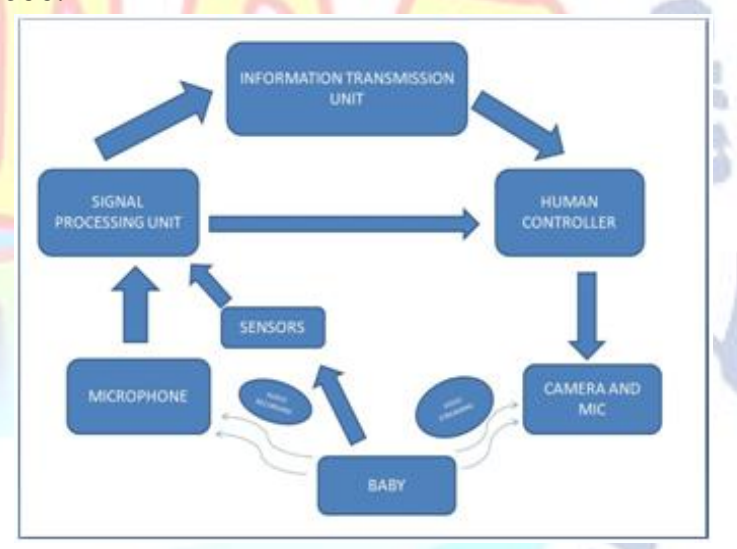

Fig. 10. Advanced Baby Monitoring System

\section{REFERENCES}

[1] Sowmyasudhan S, Manjunath S, "A Wireless Based Real-time Patient Monitoring System", International Journal of Scientific \& Engineering Research, Volume 2, Issue 11, November-2011.

[2] N.S. Joshi, R.K. Kamat, and P.K. Gaikwad, "Development of Wireless Monitoring System for Neonatal Intensive Care Unit", International Journal of Advanced Computer Research, Volume-3 Number-3 Issue-11 September-2013.

[3] V.S. Kharote-Chavan, Prof. Satyanarayana Ramchandra Rao, "Multiparameter Measurement of ICU patient using GSM and Embedded Technology", International Journal of Science and Engineering Volume1, Number 2, 2013.

[4] Ronen Luzon, "infant monitoring system", May 16, 2002. Patent No. US 2002/0057202 A1. [17]. Cynthia L_Altenhofen," BABY MONITOR SYSTEM", Mar. 28, 2000. Patent No. 6,043,747. 
[5] Kellie I. Nguyen, "infant sleeping blanket/garment for use with medical devices", Sep.17,2002, Patent No.US $6,450,168$ B1.

[6] Nur Ilyani Ramli, Mansour Youseffi, and Peter Widdop, "Design and Fabrication of a low cost heart monitor using reflectance Photoplethysmogram", World Academy of science, Engineering and Technology 082011 ,pages 417 to 418.

[7] Carsten Linti, Hansjurgen Horter, Peter Osterreicher,and Heinrich Planck, "Sensory baby vest for the monitoring of infant", International workshop on Wearable and Implantable Body Sensor Networks, BSN 2006,3-5 April 2006.

[8] Sharief F. Babiker, Liena Elrayah Abdel-Khair, and Samah M. Elbasheer, "Microcontroller Based Heart Rate Monitor using Fingertip Sensors", UofKEJ Vol. 1 Issue 2 pp. 47-51 (October 2011).

[9] Prof.K.Padmanabhan, "Microcontroller-Based Heart-Rate Meter", electronics for you ,www.efymag.com. 